\title{
Laser Direct Writing of Conductive Carbon Microelectrodes and Micro-Supercapacitor Applications
}

\author{
Akira Watanabe ${ }^{1}$ and Jinguang $\mathrm{Cai}^{2}$ \\ ${ }^{1}$ Institute of Multidisciplinary Research for Advanced Materials, Tohoku University, Japan \\ E-mail: akira.watanabe.c6@tohoku.ac.jp \\ ${ }^{2}$ Institute of Materials, China Academy of Engineering Physics, P. R. China
}

\begin{abstract}
The 3D laser direct writing of a carbon microelectrode from a carbon black aqueous dispersion was developed and the carbon electrode was applied to an in-plane micro-supercapacitor (MSC). The thickness of the electrode was increased by repeating the cycle of immersion of a glass substrate into an aqueous dispersion, lifting up the substrate from the dispersion, and galvano-scanning of $\mathrm{CO}_{2}$ laser beam on the substrate covered with the carbon black aqueous dispersion. A carbonmanganese oxide $\left(\mathrm{Mn}_{\mathrm{x}} \mathrm{O}_{\mathrm{y}}\right)$ hybrid electrode from a carbon black-manganese (II) acetate tetrahydrate aqueous dispersion was also prepared by the 3D laser direct writing. The characteristics of in-plane MSCs composed of carbon and carbon- $\mathrm{Mn}_{\mathrm{x}} \mathrm{O}_{\mathrm{y}}$ hybrid electrodes were studied. A carbon- $\mathrm{Mn}_{\mathrm{x}} \mathrm{O}_{\mathrm{y}}$ hybrid electrode showed the characteristics of a pseudo MSC and larger capacitance than a pure carbon electrode.
\end{abstract}

DOI: $10.2961 /$ jlmn.2020.02.2003

Keywords: 3D laser direct writing, conductive carbon electrode, in-plane micro-supercapacitor

\section{Introduction}

Printed electronics is one of the applications which can revolutionize the manufacturing of electronic devices by reducing the cost and energy consumption during fabrication. [1]. Inkjet printing has been widely studied as an ondemand technique in printed electronics for the development of unique flexible and transparent electronic devices. [2]. The formation of conductive micropatterns by solution processes is an important issue in printed electronics. In the case of inkjet printing, a metal nanoparticle ink as a precursor of a conductive pattern is printed on a substrate and then the substrate is heat-treated in an electronic furnace to sinter the metal nanoparticle patterns. The sintering of the metal nanoparticles is also conducted by photo-curing using a high-power xenon light source. A laser irradiation also can be applied to the sintering. The advantage of the laser direct writing using metal nanoparticle ink is that the micropatterning and sintering were conducted at same time by laser beam scanning on a metal nanoparticle film. The high resolution without any photolithography process is also advantageous in the laser direct writing. In previous papers, we have reported the laser direct writing of conductive micropatterns using $\mathrm{Ag}$ and $\mathrm{Cu}$ nanoparticles [3-8]. Our recent studies have been also focused on the formation of conductive carbon micropatterns by laser direct writing [9]. Graphene is a promising material in various technological applications because of its high electrical and thermal conductivity. Graphene oxide (GO) is a graphenefamily nanomaterial, which has the dispersibility in water and polar solvents due to the oxygen-containing groups on the graphene planes. We have reported the laser-induced reduction of GO and the formation of conductive micropatterns of reduced graphene oxide ( $\mathrm{GGO}$ ) on a flexible polymer film [10]. A graphene-based highly sensitive humidity sensor has been developed based on an $\mathrm{rGO} / \mathrm{GO} / \mathrm{rGO}$ structure. The laser direct writing on an aromatic polymer such as a polyimide (PI) also gave a conductive carbon interdigitated micropattern, which can be applied to an energy storage device such as an in-plane micro-supercapacitor (MSC) [11-13]. Micro-supercapacitor and Micro-ultracapacitor are electrochemical capacitors that have a high energy density compared to common capacitors. The advantages of laser processing in the fabrication of such devices have been reported in the pioneering studies of planar microultracapacitors $[14,15]$ The capacitance of the in-plane MSC depends on the thickness of a carbon interdigitated electrode. If a 3D structure of an interdigitated microelectrode can be fabricated by controlling the electrode thickness and shape, the enhancement of the capacitance is expected. The 3D direct laser writing of a conductive electrode is a challenging issue in energy storage devices. In this paper, we report the 3D laser direct writing of carbon electrode using a carbon black aqueous dispersion and a $\mathrm{CO}_{2}$ laser galvano-scanner combined with a PC-controlled z-axis auto-stage. A pseudo MSC consisting of carbon and redox species is an attractive devise to enhance the capacitance of MSC [16-18]. A carbon-manganese oxide $\left(\mathrm{Mn}_{\mathrm{x}} \mathrm{O}_{\mathrm{y}}\right)$ hybrid electrode was prepared by 3D laser direct writing from a carbon black-manganese (II) acetate tetrahydrate aqueous dispersion and the characteristics of MSCs were compared between those composed of carbon and carbon$\mathrm{Mn}_{\mathrm{x}} \mathrm{O}_{\mathrm{y}}$ hybrid electrode.

\section{Experimentals}

\subsection{Materials}

A carbon black with the average diameter of $24 \mathrm{~nm}$ (Mitsubishi Chemical, MA100, 2 g) was dispersed in water (20 g) using polyvinylpyrrolidone (PVP, $2 \mathrm{~g}$ ) and DEMOL 
$\mathrm{N}$ (Kao Chemicals $0.1 \mathrm{~g}$ ) as stabilizing agents under ultrasonication. A carbon black-manganese (II) acetate tetrahydrate (MnATH) dispersion was prepared by adding MnATH (1 g) to the carbon black aqueous dispersion $(20 \mathrm{~g})$. An in-plane $\mathrm{MSC}$ was prepared using $\mathrm{PVA}-\mathrm{H}_{2} \mathrm{SO}_{4}$ polymer electrolyte, which was prepared by stirring $1 \mathrm{~g}$ of poly(vinyl alcohol) (PVA) in $10 \mathrm{ml}$ of deionized water and $1 \mathrm{ml}$ of sulfuric acid $(98 \%)$.

\subsection{Laser Direct Writing}

$\mathrm{A} \mathrm{CO}_{2}$ laser galvano-scanner $(10600 \mathrm{~nm}, 20 \mathrm{~W}) \mathrm{com}-$ bined with a $\mathrm{z}$-axis auto-stage was employed for the $3 \mathrm{D}$ laser direct writing of carbon and carbon- $\mathrm{Mn}_{\mathrm{x}} \mathrm{O}_{\mathrm{y}}$ hybrid electrodes on a glass substrate. The thickness of the electrode was increased by repeating the cycle of immersion of a glass substrate into an aqueous dispersion, lifting up the substrate from the dispersion, and galvano-scanning of $\mathrm{CO}_{2}$ laser beam on the substrate covered with the carbon black aqueous dispersion. The focal length of an $\mathrm{f}-\theta$ lens was 300 $\mathrm{mm}$. The deposited electrode by the 3D laser direct writing was washed in water until the black color of the solution disappeared.

\subsection{Measurements}

The morphologies of the 3D-laser written electrodes were observed by an optical microscope (BX51, OLYMPUS). Raman spectra were measured on a microRaman spectrometer equipped with an optical microscope (Olympus BX51), a CW 532 nm DPSS laser, a Peltiercooled CCD camera (DV401, Andor Technology), and a monochromator (MS257, Oriel Instruments Co.). Surface resistivity was measured by four-point probe method.

\section{Results and discussion}

\subsection{D laser direct writing of electrodes}

The 3D laser direct writing processes in the fabrication of a carbon electrode is shown in Fig. 1, where the galvano-scanning of $\mathrm{CO}_{2}$ laser beam and z-axis scanning of a substrate were controlled by a software (Esca, BJJCZ). A glass substrate was immersed into the dispersion solution (Fig.1a) and lifted up (Fig.1b) by z-axis auto-stage. The $\mathrm{CO}_{2}$ laser beam was scanned on the substrate covered with the aqueous (Figs. 1c, d, and e), where the scan spacing of laser beam on the substrate was $40 \mu \mathrm{m}$. The $\mathrm{CO}_{2}$ laser irradiation caused the drying of the aqueous dispersion and the carbonization of carbon-PVP composite at same time. In such processes, a hybrid structure of conductive carbon electrode with a high resistive material as a redox species can be prepared if the material is soluble or dispersible into the carbon aqueous dispersion. As a redox material which improves the capacitance of an in plane MSC forming a pseudcapacitor, $\mathrm{Mn}_{\mathrm{x}} \mathrm{O}_{\mathrm{y}}$ has been studied $[18,19]$. The hybridization of $\mathrm{Mn}_{\mathrm{x}} \mathrm{O}_{\mathrm{y}}$ with a conductive carbon material is necessary because of the high resistive properties of $\mathrm{Mn}_{\mathrm{x}} \mathrm{O}_{\mathrm{y}}$. In this study, we prepared a carbon- $\mathrm{Mn}_{\mathrm{x}} \mathrm{O}_{\mathrm{y}}$ hybrid electrode by the $3 \mathrm{D}$ laser direct writing employing a carbon blackMnATH-PVP mixed aqueous dispersion. Fig. 2 shows a pure carbon electrode with the deposition cycle number of 40 (Fig. 2a) and a hybrid electrode with 20 times deposition (Fig. 2b), where the thicknesses were 4.78 and 32.0 $\mu \mathrm{m}$, respectively. The addition of MnATH enhanced the increase of the film thickness during laser deposition from the aqueous dispersion remarkably. The thicknesses of the hybrid electrodes with deposition cycle numbers of 5, 20, and 40 were $3.92,32.0$, and $78.5 \mu \mathrm{m}$, respectively. The surface resistivities of the pure carbon electrode $(4.78 \mu \mathrm{m})$ and hybrid electrodes with thicknesses of $3.92,32.0$, and $78.5 \mu \mathrm{m}$ were $1.88 \mathrm{k} \Omega, 2.95 \mathrm{M} \Omega, 2.45 \mathrm{k} \Omega$, and $2.28 \mathrm{k} \Omega$, respectively. Although the surface resistivity of the hybrid electrode was lower than the pure carbon electrode because of $\mathrm{Mn}_{\mathrm{x}} \mathrm{O}_{\mathrm{y}}$ composition, an electrode resistance can be decreased by increasing in the deposition cycle number.
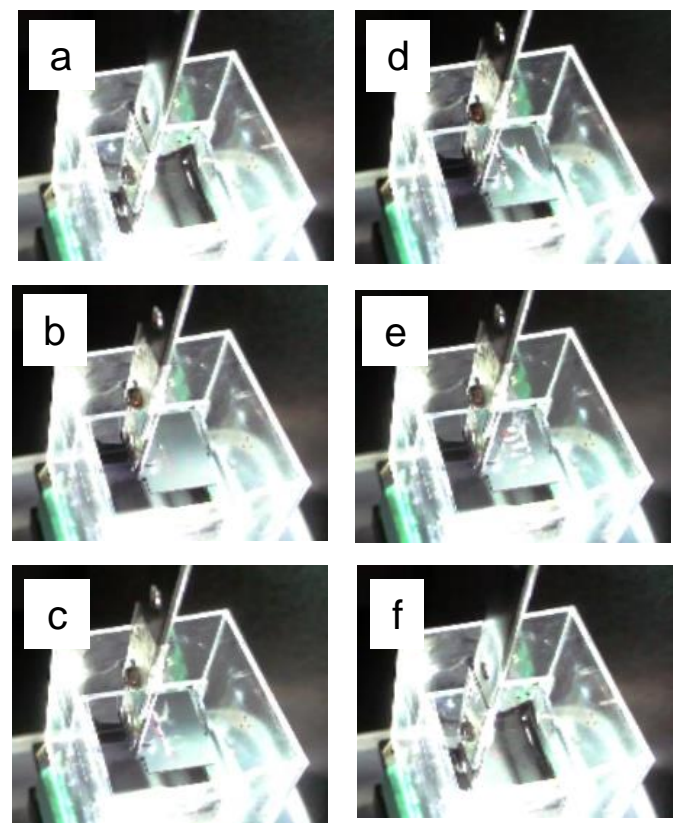

Fig.1 Photographs of 3D laser direct writing processes. (a) Immersion of a glass substrate into a carbon black-PVP aqueous dispersion by z-axis auto-stage, (b) lifting up the substrate, (c), (d), (e) galvano-scanning of $\mathrm{CO}_{2}$ laser beam, (f) immersion of the glass substrate into aqueous dispersion again.

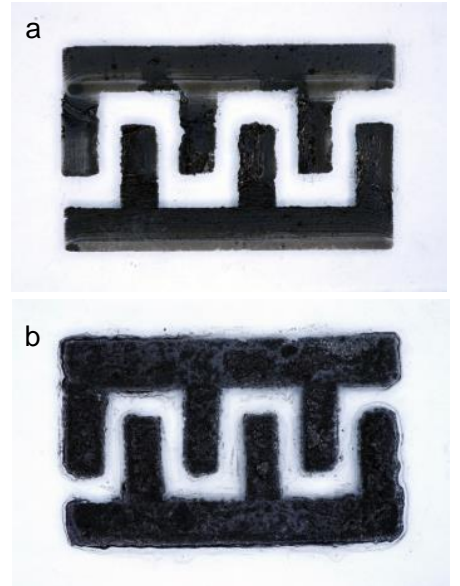

Fig.2 Photographs of carbon electrodes. (a) Pure carbon electrode with deposition cycle number of 40, carbon-manganese oxide hybrid electrode with deposition cycle number of 20 .

3.2 Morphology and chemical structure of 3D laser written electrodes 
The morphological changes with increasing in the deposition cycle number were observed by optical microscope as shown in Figs. 3 and 4. With increasing in the thickness, the surface roughness with granular structures became remarkable. The chemical structures of the carbon and carbon- $\mathrm{Mn}_{\mathrm{x}} \mathrm{O}_{\mathrm{y}}$ electrodes were investigated by micro-Raman spectroscopy.
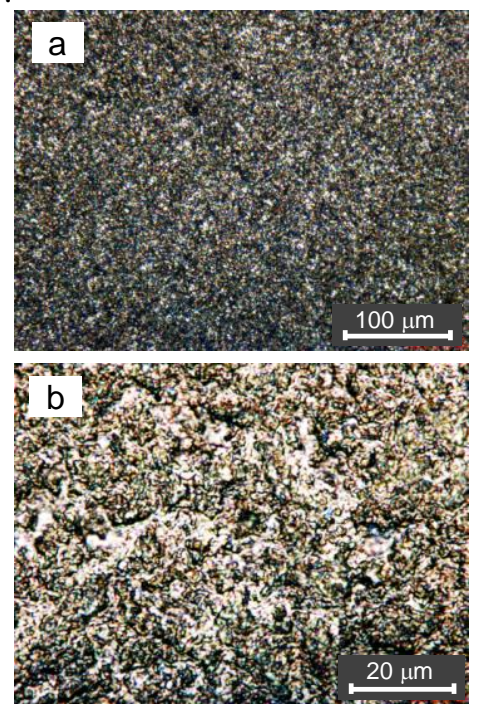

Fig.3 Optical microscope images of a carbon- $\mathrm{Mn}_{\mathrm{x}} \mathrm{O}_{\mathrm{y}}$ hybrid electrode with deposition cycle number of 5 .
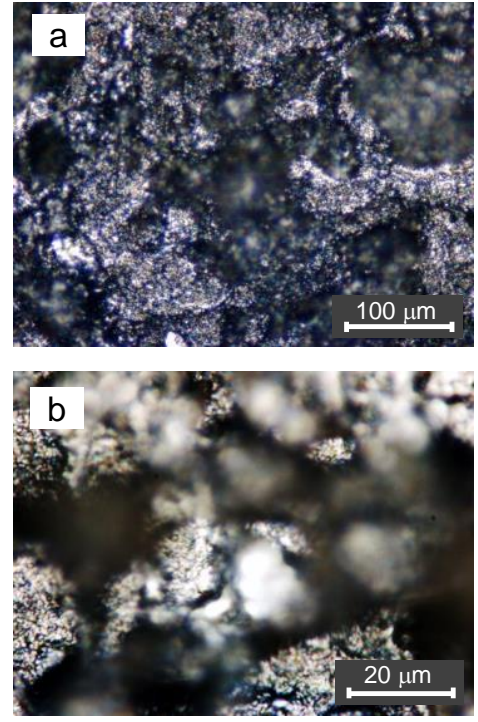

Fig.4 Optical microscope images of a carbon- $\mathrm{Mn}_{\mathrm{x}} \mathrm{O}_{\mathrm{y}}$ hybrid electrode with deposition cycle number of 20.

The Raman spectrum of the electrode prepared from a carbon black-PVP aqueous dispersion showed two broad Raman bands in the region from 1300 to $1700 \mathrm{~cm}^{-1}$ as shown in Fig. 5, which suggested the formation of amorphous carbon structure. The signals from PVP were not observed in the Raman spectrum. On the other hand, a hybrid electrode prepared from carbon black-MnATH-PVP aqueous dispersion showed new peaks as shown in Fig. 6 and the Raman spectrum changed with increasing in the deposition cycle number. The Raman spectrum of the hybrid electrode with deposition cycle number of 5 (Fig. 6a) showed Raman bands at 1100,669 , and $550 \mathrm{~cm}^{-1}$ in addition to amorphous carbon bands, which are assigned to $\mathrm{MnO}$ (II), $\mathrm{MnO}_{2}$ (IV), and $\mathrm{MnOOH}$, respectively[20-22]. With increasing in the deposition cycle number to 20 (Fig. 6 b), the Raman spectrum showed a sharp band at $649 \mathrm{~cm}^{-1}$ and weak Raman bands in the region from 300 to $400 \mathrm{~cm}^{-1}$, which can be assigned $\mathrm{Mn}_{2} \mathrm{O}_{3}$ [23]. Other remarkable feature is the increase of the Raman scattering intensity ratio of the manganese oxide band compared to carbon bands with increasing in the deposition cycle number.

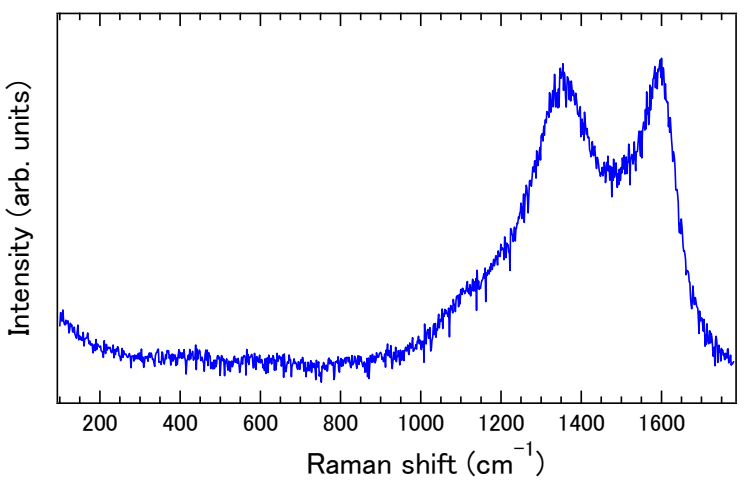

Fig.5 Raman spectrum of a pure carbon electrode with the deposition cycle number of 40 .

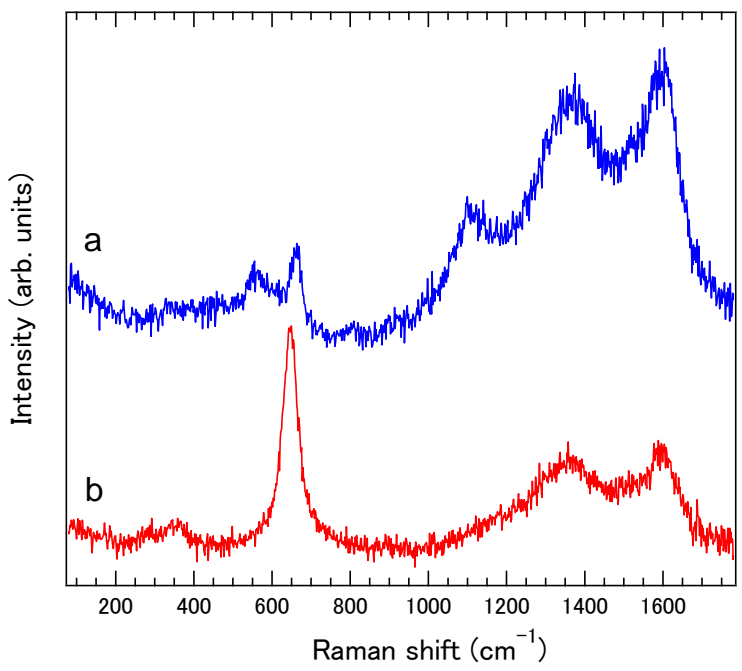

Fig.6 Raman spectrum of carbon- $\mathrm{Mn}_{\mathrm{x}} \mathrm{O}_{\mathrm{y}}$ hybrid electrodes with deposition cycle number of (a) 5 and (b) 20 .

\subsection{In plane micro-supercapacitor of 3D laser-written electrode}

In plane MSCs using 3D laser written electrodes were prepared and the cyclic voltammograms and capacitance were compared between pure carbon and carbon- $\mathrm{Mn}_{\mathrm{x}} \mathrm{O}_{\mathrm{y}}$ hybrid electrodes. The characteristics as a pseudosupercapacitor are expected to a carbon- $\mathrm{Mn}_{\mathrm{x}} \mathrm{O}_{\mathrm{y}}$ electrode. Figs. 7 and 8 show the cyclic voltammograms of a pure carbon MSC. The cyclic voltammograms showed no redox peak, which can be attributed to almost capacitive currents. The deformation of the cyclic voltammogram shape from an ideal capacitor became remarkable with increasing in 
the potential sweep rate as shown in Fig.7, which is caused by a high resistance of the electrode. Such a phenomenon became more remarkable with further increasing in the potential sweep rate as shown in Fig.8. The optimization of the 3D laser writing conditions and the composition of carbon and PVP are necessary to prepare a low resistive electrode for the performance improvement.

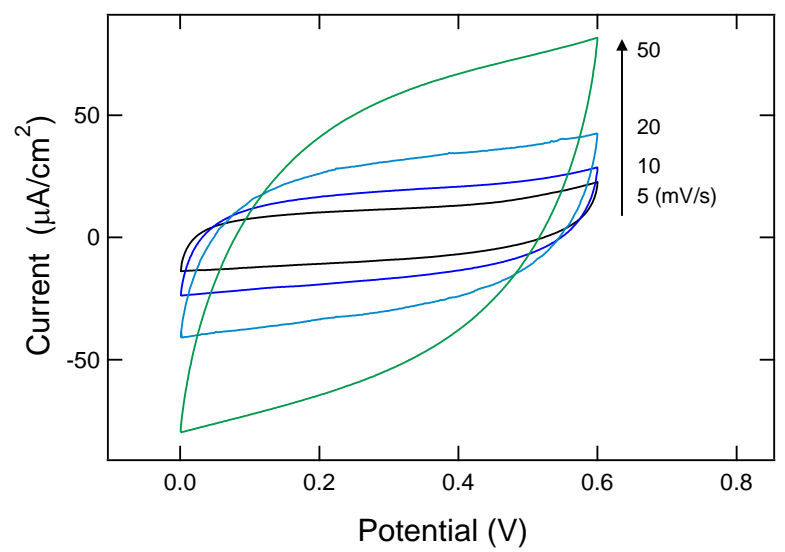

Fig.7 Cyclic voltammograms of a pure carbon MSC.

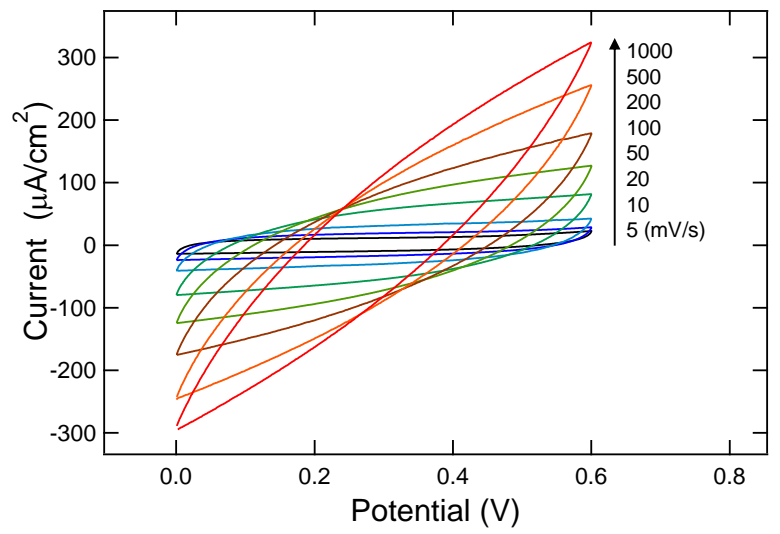

Fig.8 Cyclic voltammograms of a pure carbon MSC.

The cyclic voltammograms of a carbon- $\mathrm{Mn}_{\mathrm{x}} \mathrm{O}_{\mathrm{y}}$ hybrid MSC are shown in Figs. 9 and 10. The shapes were quite different from those of the carbon MSC, where several redox peaks were observed overlapping with capacitive current, which is the feature of a pseudo MSC. The cyclic voltammograms were stable and showed almost no change after initial changes during several cycles. Because the $\mathrm{Mn}_{\mathrm{x}} \mathrm{O}_{\mathrm{y}}$ is a complex of various manganese oxides as revealed by Raman spectroscopy, the cyclic voltammograms have several redox peaks. With increasing in the potential sweep rate, the cyclic voltammogram shape of carbon$\mathrm{Mn}_{\mathrm{x}} \mathrm{O}_{\mathrm{y}}$ hybrid MSC became almost similar to those of the carbon MSC as shown in Fig.10. Such characteristics also confirmed the assignment of the complicated peaks in cyclic voltammograms of the carbon- $\mathrm{Mn}_{\mathrm{x}} \mathrm{O}_{\mathrm{y}}$ hybrid MSC to the redox couples of $\mathrm{Mn}_{\mathrm{x}} \mathrm{O}_{\mathrm{y}}$.

The capacitances of pure and carbon- $\mathrm{Mn}_{\mathrm{x}} \mathrm{O}_{\mathrm{y}}$ MSCs are compared in Fig. 11. The capacitances of the carbon- $\mathrm{Mn}_{\mathrm{x}} \mathrm{O}_{\mathrm{y}}$ MSC was about 10 times higher than those of the carbon MSC. Such enhancement can be attributable to the pseudocapacitive characteristics of the MSC. The capacitance of the carbon- $\mathrm{Mn}_{\mathrm{x}} \mathrm{O}_{\mathrm{y}}$ hybrid $\mathrm{MSC}$ at $5 \mathrm{mV} / \mathrm{cm}^{2}$ was higher

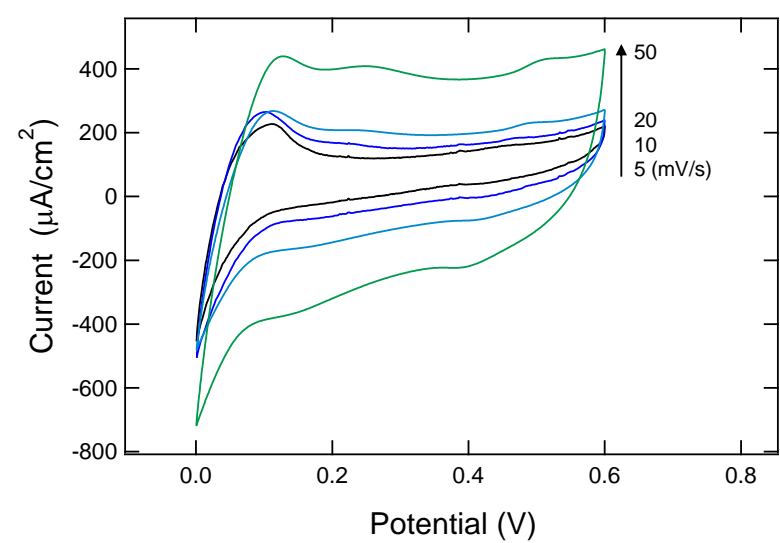

Fig.9 Cyclic voltammograms of a carbon- $\mathrm{Mn}_{\mathrm{x}} \mathrm{O}_{\mathrm{y}}$ hybrid MSC prepared with deposition cycle number of 20 .

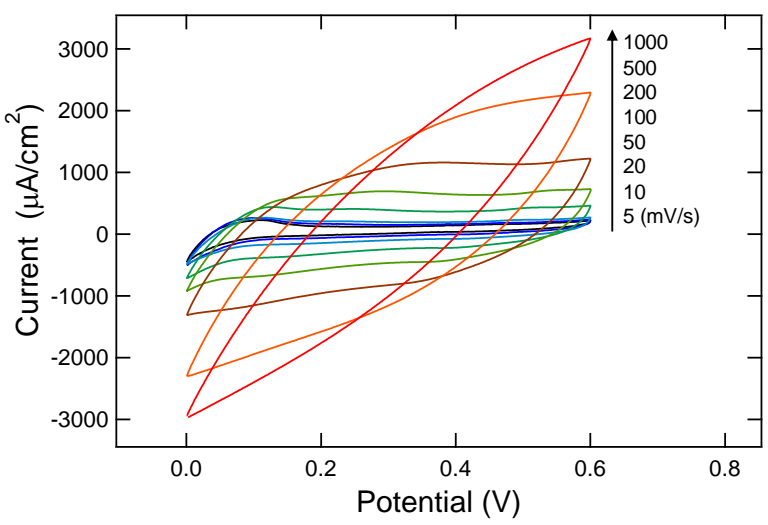

Fig.10 Cyclic voltammograms of a carbon- $\mathrm{Mn}_{\mathrm{x}} \mathrm{O}_{\mathrm{y}}$ hybrid MSC prepared with deposition cycle number of 20 .

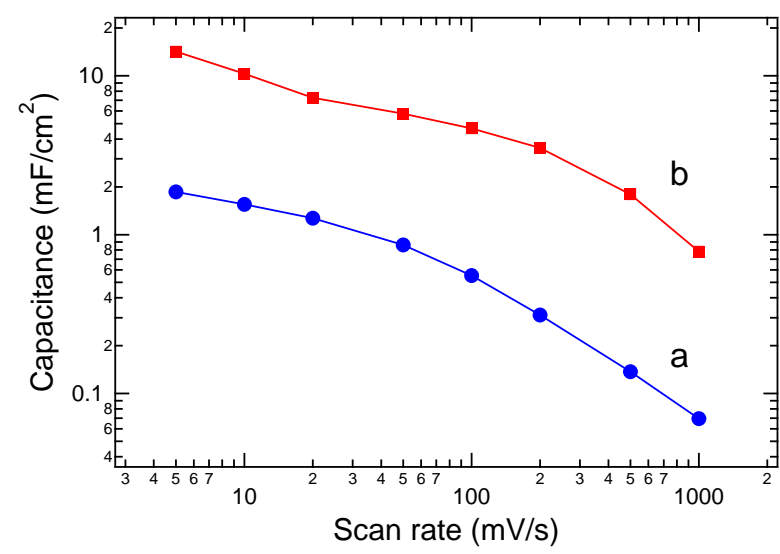

Fig.11 Capacitances of (a) pure carbon MSC with deposition cycle number of 40 and (b) carbon- $\mathrm{Mn}_{\mathrm{x}} \mathrm{O}_{\mathrm{y}}$ hybrid $\mathrm{MSC}$ with deposition cycle number of 20 .

than a high performance MSC which was prepared by the laser direct writing on a polyimide (PI) film because the thickness of the electrode can be increased by the 3D laser direct writing process in addition to the pseudo-capacitive characteristics [24]. However, the decrease of the capacitance with increasing in the potential scan rate is significant compared with the MSC prepared from laser carbonized PI because the resistance of a carbon- $\mathrm{Mn}_{\mathrm{x}} \mathrm{O}_{\mathrm{y}}$ electrode is higher than that of the laser carbonized PI. The optimization of the laser $3 \mathrm{D}$ direct writing conditions is necessary to 
improve the performance of a carbon- $\mathrm{Mn}_{\mathrm{x}} \mathrm{O}_{\mathrm{y}}$ hybrid MSC in the higher scan rates. The optimization of the polymer electrolyte for the carbon- $\mathrm{Mn}_{\mathrm{x}} \mathrm{O}_{\mathrm{y}}$ electrode is also important issue to enhance the capacitance because the potential sweep range was limited below $0.6 \mathrm{~V}$ to avoid the degradation of $\mathrm{Mn}_{\mathrm{x}} \mathrm{O}_{\mathrm{y}}$ due to $\mathrm{H}_{2} \mathrm{SO}_{4}$ containing electrolyte.

\section{Conclusion}

The 3D laser direct writing technique for the preparation of carbon electrode was developed by combining $\mathrm{CO}_{2}$ laser galvano-scanner and z-axis auto-stage, which is the direct deposition process of a carbon material from a liquid dispersion to a substrate. One of the advantages of the 3D laser direct writing is that various kinds of materials can be hybridized by mixing them with carbon. Such a feature can be applicable to prepare a carbon hybrid electrode for pseudo MSC. The increase of the electrode thickness by the 3D laser deposition process is also advantageous to enhance the capacitance of a MSC. A carbon- $\mathrm{Mn}_{\mathrm{x}} \mathrm{O}_{\mathrm{y}}$ hybrid MSC prepared by the 3D laser direct writing showed the characteristics as a pseudo MSC and the higher capacitance than a carbon MSC.

\section{Acknowledgments}

This work was partially supported by JSPS KAKENHI Grant Number JP15H04132, JSPS KAKENHI Grant Number JP19H05356, National Natural Science Foundation of China (No. 21603201), and China Academy of Engineering Physics (item no. TP201302-3).

\section{References}

[1] K. Fukuda and T. Someya: Adv. Mater., 29, (2017) 1602736 .

[2] M. Gao, L. Li and Y. Song: J. Mater. Chem. C, 5, (2017) 2971.

[3] A. Watanabe and J. Cai: J. Photopolym. Sci. Tec., 30, (2017) 341.

[4] G. Qin, L. Fan and A. Watanabe: J. Mater. Process. Technol., 227, (2016) 16.

[5] M. Aminuzzaman, A. Watanabe and T. Miyashita: J. Electron. Mater., 44, (2015) 4811.

[6] G. Qin, A. Watanabe, H. Tsukamoto and T. Yonezawa: Jpn. J. Appl. Phys., 53, (2014) 096501.
[7] G. Qin and A. Watanabe: J. Nanopart. Res., 16, (2014) 2684.

[8] M. Aminuzzaman, A. Watanabe and T. Miyashita: J. Nanopart. Res., 12, (2010) 931.

[9] J. Cai, C. Lv and A. Watanabe: ACS Appl. Mater Interfaces, 10, (2018) 915.

[10]J. Cai, C. Lv, E. Aoyagi, S. Ogawa and A. Watanabe: ACS Appl. Mater. Interfaces, 10, (2018) 23987.

[11]J. Cai, C. Lv and A. Watanabe: RSC Adv., 7, (2017) 415.

[12]J. Cai, C. Lv and A. Watanabe: Nano Energy, 30, (2016) 790.

[13]J. Cai, C. Lv and A. Watanabe: J. Mater. Chem. A, 4, (2016) 1671

[14]C. B. Arnold, R. C. Wartena, K. E. Swider-Lyons and Alberto Pique: J. Electrochem. Soc., 150, (2003) A571.

[15]C. B. Arnold, R. C. Wartena, B. Pratap, K. E. SwiderLyons and Alberto Pique: Proc. SPIE, Photon Processing in Microelectronics and Photonics, 4637, (2002) 353.

[16]L. Li, Z. Lou, W. Han, D. Chen, K. Jiang and G. Shen: Adv. Mater. Technol., 2, (2017) 1600282.

[17]L. Sun, X. Wang, K. Zhang, J. Zou and Q. Zhang: Nano Energy, 22, (2016) 11.

[18]L. Li, J. Zhang, Z. Peng, Y. Li, C. Gao, Y. Ji, R. Ye, N.D. Kim, Q. Zhong, Y. Yang, H. Fei, G. Ruan and J.M. Tour: Adv. Mater., 28, (2016) 838

[19]Z. Zhang, F. Xiao, L. Qian, J. Xiao, S. Wang and Y. Liu: Adv. Energy Mater., 4, (2014) 1400064.

[20]Y. Wang, H. Guan, S. Du and Y. Wang: RSC Adv., 5, (2015) 88979-88988

[21]T.-H. Wu, D. Hesp, V. Dhanak, C. Collins, F. Braga, L.J. Hardwick and C.-C. Hu: J. Mater. Chem. A, 3, (2015) 12786.

[22]N. Mironova-Ulmanea, A. Kuzmin and M. Grube: J. Allys Compd. 480, (2009) 97.

[23]A. Ramírez, P. Hillebrand, D. Stellmach, M.M. May, P. Bogdanoff and S. Fiechter: J. Phys. Chem. C, 118, (2014) 14073.

[24]J. Lin, Z. Peng, Y. Liu, F. Ruiz-Zepeda, R. Ye, E.L.G. Samuel, M.J. Yacaman, B.I. Yakobson and J.M. Tour: Nat. Commun., 5, (2014) 5714.

(Received: May 21, 2019, Accepted: July 28, 2020) 